\title{
TECHNIQUES
}

\section{A novel method for transcatheter closure of atrial septal defect within an aneurysm of the fossa ovalis: double sheath technique}

\author{
M Peuster, R Kaulitz, G Hausdorf
}

Department of Pediatric Cardiology and Pediatric Intensive Care, Hannover Medical School, Carl

Neuberg Straße 1, D-30625 Hannover, Germany

M Peuster

R Kaulitz

G Hausdorf

Correspondence to: Dr Peuster

peuster.matthias@

MH-Hannover.de

Accepted 6 September 2000

\begin{abstract}
An aneurysm of the fossa ovalis is frequently associated with an atrial septal defect. Intervention with transcatheter closure of such defects may be difficult since it bears the risk of inadvertent deployment of the device with both umbrellas in the right or left atrium. A novel technique for the closure of such multiperforated aneurysms of the fossa ovalis, which allows central positioning and controlled deployment of the device, is reported.

(Heart 2000;84:e14)
\end{abstract}

Keywords: fossa ovalis; aneurysm; atrial septal defect; transcatheter closure

To facilitate central crossing of the multiperforated aneurysm of the fossa ovalis, a balloon tipped catheter (Berman Wedge) was filled with diluted contrast medium within the right atrium and advanced under echocardiographic guidance into the aneurysm of the fossa ovalis, thus centring the end hole of the catheter within the aneurysm (fig 1A). A guidewire was advanced through the catheter into the left atrium; the balloon was deflated and also advanced into the left atrium and further into the left upper pulmonary vein. Transoesophageal echocardiography confirmed that the catheter crossed the centre of the multiperforated aneurysm of the fossa ovalis. During sizing of the defect, echocardiography demonstrated that the redundant tissue of the aneurysm was pushed into the left upper pulmonary vein.

Inadvertent deployment of both umbrellas of the CardioSeal device in the left atrium was avoided by using a double sheath technique. A $23 \mathrm{~mm}$ CardioSeal device was front-loaded into a 9 French long sheath which was preshaped like an NIH catheter using hot vapour. An 11 French long sheath was shortened, so that it was $6 \mathrm{~cm}$ shorter than the 9 French introducer sheath, and pre-shaped using hot vapour. The 11 French long sheath was advanced into the left upper pulmonary vein over the 0.035 inch guidewire. The dilator and guidewire were retrieved and the 9 French long sheath, which had been preloaded with the $23 \mathrm{~mm}$ CardioSeal device, was advanced to the tip of the 11 French sheath. The left umbrella of the device was opened within the left upper pulmonary vein, to assure its position on the left atrial side of the floppy aneurysm of the fossa ovalis (fig 1B). Thereafter, both sheaths
A

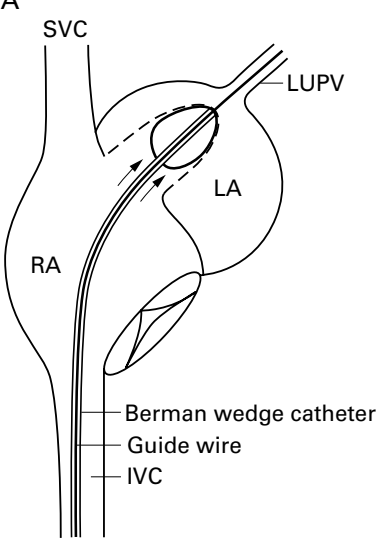

B

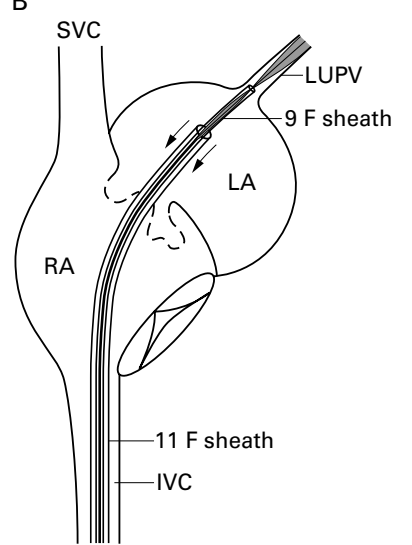

C

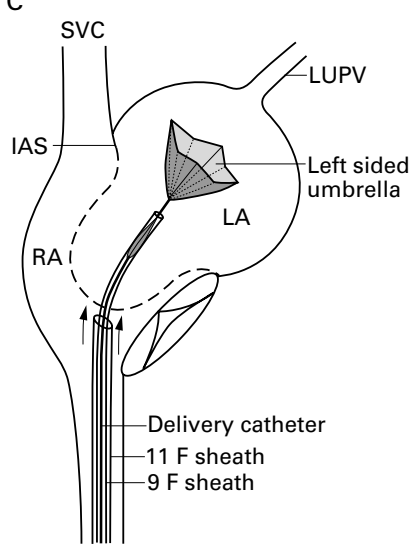

D

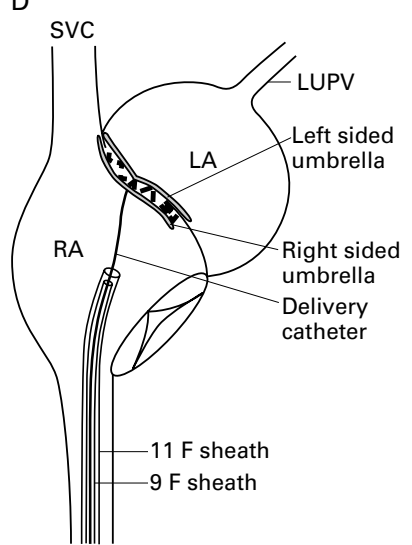

Figure 1 (A) The Berman wedge catheter is filled with dilute contrast medium and pushed centrally into the aneurysmatic atrial septal defect. The guidewire is then advanced into the left upper pulmonary vein. (B) The umbrella and delivery system are manipulated into a 9 French sheath and loaded into an 11 French sheath. The 11 French long sheath is advanced across the aneurysm defect and the left atrial umbrella is opened in the left upper pulmonary vein. (C) The left atrial umbrella is opened. The left atrial sheath is pulled back into the inferior vena cava and readvanced towards the interatrial septum to ascertain its right atrial position. (D) Both arms of the CardioSeal device are opened with the aneurysm interatrial septum being trapped between the right atrial and left atrial umbrella. SVC, superior vena cava; IVC, inferior vena cava; LUPV, left upper pulmonary vein; RA, right atrium; $L A$, left atrium; $I A S$, interatrial septum. 
were pulled back under echocardiographic guidance until the left atrial arms of the CardioSeal device were in contact with the interatrial septum. The 11 French long sheath was pulled back under echocardiographic guidance over the 9 French long sheath into the inferior vena cava, to assure that the tip of the 11 French long sheath was on the right side of the interatrial septum. The 11 French long sheath was then re-advanced towards the CardioSeal device to push the aneurysmatic tissue to the centre of the device (fig 1C). Approximation of the aneurysm tissue to the (opened) left atrial umbrella was confirmed by hand injections with contrast through the 11 French sheath and by echocardiography. The right umbrella of the CardioSeal device was opened into the 11 French sheath by pulling back the 9 French sheath. Echocardiography demonstrated that the aneurysmatic tissue was still kept in position by the 11 French long sheath. To deploy the right atrial arms the 11 French long sheath was pulled back. The right atrial umbrella was opened on the right side of the aneurysmatic fossa ovalis, resulting in complete closure of the multiperforated aneurysm of the fossa ovalis. The aneurysmatic tissue was caught within the device (fig 1D). Echocardiography six months after the procedure showed complete occlusion of the multiperforated aneurysm of the fossa ovalis and capture of the aneurysmatic tissue between both umbrellas of the device, with no residual shunting. 